Published in final edited form as:

Anaesthesia. 2005 February ; 60(2): 113-117.

\title{
Placement of Intubating Laryngeal Mask Airway Is Easier than Placement of Laryngeal Tube during Manual In-Line Stabilisation of The Neck
}

\author{
R. Komatsu ${ }^{1}$, O. Nagata ${ }^{2}$, K. Kamata ${ }^{3}$, K. Yamagata ${ }^{4}$, D.I. Sessler ${ }^{5}$, and M. Ozaki ${ }^{6}$ \\ 1 Clinical instructor, Department of Anesthesiology, Tokyo Women's Medical University; Research \\ Fellow, Outcomes Research ${ }^{T M}$ Institute, University of Louisville.
}

2 Assistant Professor, Department of Anesthesiology, Tokyo Women's Medical University.

3Clinical instructor, Department of Anesthesiology, Tokyo Women's Medical University.

4Clinical instructor, Department of Anesthesiology, Tokyo Women's Medical University.

5 Vice Dean for Research, Associate Vice President Health Affairs, Director Outcomes $R_{\text {ESEARCH }}{ }^{T M}$ Institute, Lolita and Samuel Weakley Distinguished University Research Chair, Professor of Anesthesiology \& Perioperative Medicine and Pharmacology, University of Louisville.

6Professor and Chair, Department of Anesthesiology, Tokyo Women's Medical University.

\section{Summary}

\begin{abstract}
We compared the usefulness of the laryngeal tube (LT) with the intubating laryngeal mask airway (ILMA) in 51 patients whose necks were stabilised by manual in-line traction. After induction of anaesthesia and neuromuscular block, the LT and ILMA were inserted consecutively in a randomised, crossover design. During pressure-controlled ventilation $\left(20 \mathrm{cmH}_{2} \mathrm{O}\right.$ inspiratory pressure), we measured insertion attempts, time to establish positive-pressure ventilation, tidal volume, gastric insufflation, and minimum airway pressure at which gas leaked around the cuff. Data were compared using Wilcoxon signed-rank tests; $P<0.05$ was considered significant. Insertion was more difficult with the LT (successful at first attempt in 16 patients) than with the ILMA (successful at first attempt in 42 patients, $P<0.0001)$. Time required for insertion was longer for the LT (28 [23-35] sec, median [interquartile range]) than the ILMA (20 [15-25] sec, $P=0.0009)$. Tidal volume was less for the LT (440 [290-670] ml) than the ILMA. (630 [440-750] ml, $P=0.013)$. Minimum airway pressure at which gas leak occurred and incidence of gastric insufflation were similar with two devices. In patients whose necks were stabilised with manual in-line traction, insertion of the ILMA was easier and quicker than insertion of the LT and tidal volume was greater with the ILMA than the LT.
\end{abstract}

\section{Keywords}

Anaesthesia; equipment; airway; intubating laryngeal mask airway; laryngeal tube; cervical spine immobilization

In patients with unstable cervical spine, tracheal intubation may be required with the patient's occiput placed directly on the trolley and the head and neck stabilised (manual in-line stabilisation). Both tracheal intubation and ventilation via a facemask are often difficult in this

Send correspondence to Dr. Komatsu, Outcomes ResEarch TM Institute, 501 East Broadway, Suite 210, Louisville, KY 40202. Tel: 1-502-562-6820, Fax: 1-502-852-2610, e-mail:r0koma01@gwise.louisville.edu, on the world wide web: www.or.org..

Dr. Ryu Komatsu, 501 E. Broadway, Suite 210, Louisville, KY 40202, USA, Phone: 1-502-562-6820, Fax: 1-502-852-2610, e-

mail:r0koma01@gwise.louisville.edu 
position [1]. The intubating laryngeal mask airway (ILMA, Laryngeal Mask Company, Henley-on-Thames, UK) has been shown to effectively secure the airway, even when the neck is protected by in-line stabilisation [2,3]. However, the ILMA exerts considerably high pressures against the cervical vertebrae [4] and Keller et al. (4) recommend that the ILMA should only be used in unstable cervical spine if difficulties are anticipated or encountered with established techniques.

The laryngeal tube (LT, VBM, Medizintechnik, Sulz, Germany) (Fig. 1) is an extra-glottic airway device, which consists of an airway tube with a small balloon cuff attached at the tip (distal cuff), and larger balloon cuff at the middle part of the tube (proximal cuff). A patient may be in neutral or in sniffing position during insertion [5]. When positioned with the recommended intracuff volume, the pressures exerted by the cuffs of the LT against the pharyngeal mucosa are smaller than that exerted by the ILMA against the cervical spine [6]. We can thus assume that the amount of pressure exerted by the LT against the cervical vertebrae is less than that exerted by the ILMA. Furthermore, fiberoptic tracheal intubation through the LT is possible by the method of Genzwuerker et al. (7). A flexible fiberoptic bronchoscope with a mounted tube exchanger is inserted into trachea through laryngeal aperture of the LT. After removal of fiberoptic bronchoscope and the LT, an endotracheal tube is pushed into the trachea by mounting it on the tube exchanger, which is left in the trachea [7]. The LT may thus be useful for airway management in patients whose head and neck movements are restricted.

The aim of this study was to compare placement and performance characteristics of the LT with the ILMA during positive pressure ventilation of the lungs of patients whose heads and necks were stabilised by manual, in-line traction (8) to simulate airway management of a patient with suspected cervical spine injury.

\section{Methods}

With approval of the Human Research Committee at Tokyo Women's Medical University and informed written consent from all patients, we studied 51 patients who were undergoing elective surgical procedures under general anaesthesia. Exclusion criteria included age $<18$ $\mathrm{yr}$; ASA status 3 or greater; any pathology of the cervical spine, respiratory or upper alimentary tracts; increased risk of pulmonary aspiration; and anticipated airway difficulties (e.g., Mallampati grade 4, thyromental distance less than $6 \mathrm{~cm}$, or interincisor distance less than 3 $\mathrm{cm})$.

\section{Protocol}

Anaesthesia was induced with fentanyl $2 \mu \mathrm{g} \cdot \mathrm{kg}^{-1}$ and propofol $2 \mathrm{mg} \cdot \mathrm{kg}^{-1}$. After confirmation of adequate facemask ventilation, vecuronium $0.1 \mathrm{mg} / \mathrm{kg}$ was given for muscle relaxation. Anaesthesia was maintained with $2 \%$ sevoflurane in oxygen. Pillows were removed and the patient's head and neck were stabilised by manual, in-line traction by an assistant grasping patient's mastoid processes to keep neutral head and neck position (8) to simulate airway management of a patient with suspected cervical spine injury.

In a randomised crossover fashion, the LT and ILMA were positioned consecutively. Randomisation was based on computer-generated codes that were maintained in sequentially numbered opaque envelopes.

For the LT, we used size 3 for patients $<155 \mathrm{~cm}$ tall and size 4 for patients $\geq 155 \mathrm{~cm}$ (9). The LT was inserted by placing the tip against the hard palate behind the upper incisors and sliding the device down the centre of the mouth until resistance was felt or the second bold black line on the tube was positioned between the upper and lower teeth. The cuffs were inflated with air (size 3: $60 \mathrm{ml}$, size 4: $80 \mathrm{ml}$ ). We confirmed ventilation by connecting the breathing circuit to 
the device and gently squeezing the reservoir bag and observing the presence of end-tidal carbon dioxide waveforms and chest movement. If it was not possible to ventilate the lungs, we adjusted the position of the LT by gently pushing or pulling it. Adequacy of ventilation was then re-assessed. After confirmation of ventilation, intra-cuff pressure was adjusted 60 to 70 $\mathrm{cm} \mathrm{H}_{2} \mathrm{O}$ with a cuff inflator (VBM, Medizintechnik, Sulz, Germany).

For the ILMA, we used size 3 for patients $<160 \mathrm{~cm}$ tall, size 4 for patients between 160-170 $\mathrm{cm}$, and size 5 for patients $>170 \mathrm{~cm}(10)$. The ILMA was inserted using the one-handed rotational technique [8]. The cuff was inflated with air (size 3: $20 \mathrm{ml}$, size 4: $30 \mathrm{ml}$, size 5: 40 $\mathrm{ml}$ ). After confirmation of adequate ventilation with the same method as the LT, intra-cuff pressure was adjusted to between 60 and $70 \mathrm{~cm} \mathrm{H}_{2} \mathrm{O}$.

When initial insertion of or ventilation with an airway device failed, up to two reinsertion attempts were permitted. If placement failed after a total of three attempts, the alternative study device was tried (if the failed device was the first device tested) or the patient's airway was secured as clinically needed (if the failed device was the second device tested). All LT and ILMA insertion procedures were performed by a single anaesthesiologist (RK), who had experience with approximately 150 ILMA insertions and $50 \mathrm{LT}$ insertions before beginning the study. Pressure-controlled ventilation with $100 \%$ oxygen at a fresh gas flow of $5 \mathrm{~L} /$ minute was initiated at an inspiratory pressure at $18 \mathrm{~cm} \mathrm{H}_{2} \mathrm{O}$ using Aestiva/5 7100 anaesthesia machine (Aestiva/5 7100, Datex-Ohmeda, Helsinki, Finland). The inspiratory/expiratory ratio was 1:2, and the respiratory rate was 12 breaths per minute.

The peak inspiratory pressure associated with oropharyngeal gas leak was determined with an "up-and-down" technique. If there was no oropharyngeal gas leak, inspiratory pressure was increased by $1 \mathrm{~cm} \mathrm{H}_{2} \mathrm{O}$ each breath to a maximum of $30 \mathrm{~cm} \mathrm{H}_{2} \mathrm{O}$ by using pressure controlled ventilation mode (Aestiva/5 7100, Datex-Ohmeda, Helsinki, Finland); in contrast, inspiratory pressure was reduced $1 \mathrm{~cm} \mathrm{H}_{2} \mathrm{O}$ each breath when a gas leak was identified. This procedure was repeated for each device. The minimum inspiratory pressure that consistently caused an oropharyngeal gas leak around the test device was recorded. Subsequently, the inspiratory pressure was set at $20 \mathrm{~cm} \mathrm{H}_{2} \mathrm{O}$, and the expired tidal volume was measured as an average of 10 consecutive breaths. Airways management for the remainder of the case was at the discretion of the attending anaesthesiologist.

\section{Measurements}

Each patient's Samsoon and Young (12) modification of the Mallampati airway classification, interincisor distance, thyromental distance, and sternomental distance were measured preoperatively in upright position. The time required for successful insertion of the devices (defined as the time from picking up the device to the confirmation of capnographic trace through the device), the number of insertion attempts until successful ventilation was established, and the number of patients successfully ventilated within three insertion attempts were recorded. Successful ventilation was defined as existence of chest expansion during inspiratory phase and regular rectangular capnographic waveform during expiratory phase with or without air leakage around the device. The presence or absence of gastric insufflation was also recorded at the inspiratory pressure of $20 \mathrm{~cm} \mathrm{H}_{2} \mathrm{O}$ by auscultation over the epigastrium [9].

\section{Statistics}

Our primary goal was to compare the success rate to establish positive pressure ventilation with each device at the first insertion. This endpoint was thus used to estimate the number of patients required for our study. Assuming the success rate at the first insertion of the ILMA with neck stabilisation would be roughly the same as that in patients without neck stabilisation, 
which is approximately $90 \%$ [10], we decided that a 20 percentage point difference in success rate at the first insertion attempt would be clinically important. Since this is a crossover study with a dichotomous outcome, we used a two-sided McNemar test (alpha $=0.05)$. Assuming $30 \%$ of participants would have discordant results between ILMA and LT at the first insertion, fifty patients were required to detect a 20 percent point difference in the success rate with $80 \%$ power.

The Wilcoxon signed rank test was used to compare the success rate of first insertion attempt, the success rate within three insertion attempts, number of insertion attempts required to obtain adequate ventilation, the time required for successful insertion, oropharyngeal gas leak pressure during pressure-controlled ventilation, expired tidal volume obtained with pressure-controlled ventilation at an inspiratory pressure of $20 \mathrm{~cm} \mathrm{H}_{2} \mathrm{O}$, and presence or absence of gastric insufflation at inspiratory pressure of $20 \mathrm{~cm} \mathrm{H}_{2} \mathrm{O}$. Statistical analysis was performed using StatView version 5.0 (SAS Institute Inc. Cary, NC, USA) and Sample Power 2.0 (SPSS, Chicago, IL, USA). Values are expressed as mean (SD) or median [interquartile range] as appropriate; $P<0.05$ was considered statistically significant.

\section{Results}

Demographic and airway assessment data are shown in Table 1. Among the 51 patients, ILMA was used as the first device on 25 patients whereas the LT was used first on 26.

Success rate at first attempt to provide adequate ventilation was lower for the LT than the ILMA (16 vs. 42 attempts; $P<0.0001$ ) (Table 2). More insertion attempts were required to obtain adequate ventilation $(P<0.001)$, the time required for successful insertion was longer (28 [23$35]$ sec vs. 20 [15-25] sec; $P=0.001)$, and the success rate for obtaining adequate ventilation within three insertion attempts was lower for the LT than the ILMA (43 vs. 51 patients; $P=$ 0.005) (median [interquartile range]). Of the 51 patients studied, both devices were successful at first attempt in 12; the ILMA was successful at first attempt and the LT failed at first attempt in 30; the LT was successful at first attempt and the ILMA failed at first attempt in 4; and both devices failed at first attempt in 5 .

Expired tidal volume obtained during pressure-controlled ventilation at an inspiratory pressure of $20 \mathrm{~cm} \mathrm{H}_{2} \mathrm{O}$ was less with the LT than the ILMA (440 [290-670] $\mathrm{ml} v s$. 630 [440-750] ml; $P=0.013)$. The inspiratory pressures at which oropharyngeal gas leaked during pressurecontrolled ventilation were similar (24 [21-30] $\mathrm{cmH}_{2} \mathrm{O}$ for LT vs. 27 [20-30] $\mathrm{cmH}_{2} \mathrm{O}$ for ILMA; $P=0.44)$, as was the occurrence of gastric insufflation during pressure-controlled ventilation ( 2 of 43 for LT $v s .5$ of 51 for ILMA; $P=0.32$, Table 2).

\section{Discussion}

We found that the ILMA offers advantages compared with the LT in most technical aspects of airway management. These include greater first insertion success rate, better overall insertion success rate within three attempts, shorter insertion time, and greater expired tidal volume during pressure-controlled ventilation.

Our insertion success rate for the ILMA was in accordance with that previously reported in patients whose necks were stabilised with manual in-line traction $[2,3]$. The success rate and the time required for insertion were also similar to those in our preliminary study without neck stabilisation [11]. Our current results thus confirmed the ease with which the ILMA can be inserted in the patients with stabilised necks. Our first insertion and overall insertion success rate of the LT were lower than those reported for patients in sniffing position during insertion [12-16]. In most of our patients, the failure was associated with the difficulty of the getting the LT tip to slide on the posterior pharyngeal wall and advance into the hypopharynx. It is 
likely that this occurred because of the incomplete alignment of the oral cavity and laryngopharynx axes with the neck being fixed in the neutral position. Although Dorges et al. [17] reported $100 \%$ LT insertion success in the airways of patients with the neck in neutral position, manipulation of the head and neck might have increased their success rate because they did not stabilise the neck during insertion.

The oropharyngeal gas leak pressures did not differ significantly between the devices, and the values recorded for the devices were similar to those previously reported [12-16,18]. We found that expired tidal volume was smaller for the LT than the ILMA during pressure-controlled ventilation. This presumably results since resistance to gas flow through the device is greater because the LT is narrower and has a smaller distal aperture. Another potential explanation is increased resistance between the distal aperture of the LT and the trachea, as a consequence of LT malposition. For example, Cook et al. [15] reported that part of laryngeal inlet was visible fibre-optically from ventilation orifices only in $42 \%$ of the patients with the LT using the same intra-cuff pressure range as in our patients, although the laryngeal inlet was visible in 50\% and $70 \%$ of the patients with intra-cuff pressure of 40 and $86 \mathrm{~cm} \mathrm{H}_{2} \mathrm{O}$, respectively, with the ILMA [15]. Brimacombe et al. [19] reported a tidal volume during pressure-controlled ventilation similar to ours, although they employed an inspiratory pressure of $17 \mathrm{~cm} \mathrm{H}_{2} \mathrm{O}$. They also reported the tidal volume obtained in the position without a pillow (similar to our study conditions) was approximately $70 \mathrm{ml}$ less than that obtained with a standard pillow during pressure controlled ventilation. This reduction in tidal volume is likely to be caused by rigidity of the LT, which prevents the device adjusting to the change in shape of the upper airway. Neutral position without a pillow thus appears to be a sub-optimal position for LT ventilation.

Our study had several limitations. First, although the investigator who inserted the airways was experienced with both devices, he had three times more experience with the ILMA than with the LT. This may have given an advantage to insertion of the ILMA. However, there was no evidence of skill acquisition with the LT during the trial ( 1 failed insertion occurred in first 10 patients; 3 failed insertion occurred in the last 10 patients). Second, we did not determine the anatomic position of either airway device and it is possible that the LT was malpositioned more frequently. However, fiberoptic assessment of LT position is not routine in the clinical setting.

We conclude that the ILMA offers advantages over the LT in most technical aspects of airway management in patients undergoing pressure-controlled ventilation whose necks are stabilised in neutral position with the manual in-line method, although possible risk of neurological deterioration by the pressure caused by the ILMA against cervical spine must be considered in patients with unstable cervical spine (20).

\section{Acknowledgements}

Supported by NIH Grant GM 061655 (Bethesda, MD), the Gheens Foundation (Louisville, KY), the Joseph Drown Foundation (Los Angeles, CA), and the Commonwealth of Kentucky Research Challenge Trust Fund (Louisville, $\mathrm{KY})$.

\section{References}

1. Pennant JH, Pace NA, Gajraj NM. Role of laryngeal mask airway in the immobile cervical spine. Journal of Clinical Anesthesia 1993;5:226-30. [PubMed: 8318242]

2. Asai T, Murao K, Tsutsumi T, Shingu K. Ease of tracheal intubation through the intubating laryngeal mask during manual in-line head and neck stabilisation. Anaesthesia 2000;55:82-5. [PubMed: 10594437]

3. Asai T, Wagle AU, Stacey M. Placement of the intubating laryngeal mask is easier than the laryngeal mask during manual in-line neck stabilization. British Journal of Anaesthesia 1999;82:712-4. [PubMed: 10536548] 
4. Keller C, Brimacombe J, Keller K. Pressures exerted against the cervical vertebrae by the standard and intubating laryngeal mask airways: a randomized, controlled, cross-over study in fresh cadavers. Anesthesia and Analgesia 1999;89:1296-300. [PubMed: 10553855]

5. Anonymous., Instruction for use. Laryngeal tube (LT). 2000, Sulz, Germany: VBM Medizintechnik Gmbh.

6. Brimacombe J, Keller C, Roth W, Loeckinger A. Large cuff volumes impede posterior pharyngeal mucosal perfusion with the laryngeal tube airway. Canadian Journal of Anaesthesia 2002;49:1084-7. [PubMed: 12477684]

7. Genzwuerker H, Vollmer T, Ellinger K. Fibreoptic tracheal intubation after placement of the laryngeal tube. British Journal of Anaesthesia 2002;89:733-8. [PubMed: 12393772]

8. Brain AIJ, LMA-FastrachTM Instruction Manual 1997: The Laryngeal Mask Company Ltd,.

9. Brimacombe J, Keller C, Kurian S, Myles J. Reliability of epigastric auscultation to detect gastric insufflation. British Journal of Anaesthesia 2002;88:127-9. [PubMed: 11881867]

10. Kihara S, Yaguchi Y, Watanabe S, Brimacombe J, Taguchi N, Yamasaki Y. Haemodynamic responses to the intubating laryngeal mask and timing of removal. Eur J Anaesthesiol 2000;17:744-50. [PubMed: 11122312]

11. Komatsu R, Nagata O, Sessler DI, and Ozaki M. The intubating laryngeal mask airway facilitates tracheal intubation in the lateral position. Anesthesia and Analgesia 2004 in press.

12. Asai T, Murao K, Shingu K. Efficacy of the laryngeal tube during intermittent positive pressure ventilation. Anaesthesia 2000;55:1099-102. [PubMed: 11069337]

13. Asai T, Kawashima A, Hidaka I, Kawachi S. The laryngeal tube compared with the laryngeal mask: insertion, gas leak pressure and gastric insufflation. British Journal of Anaesthesia 2002;89:729-32. [PubMed: 12393771]

14. Asai T, Shingu K, Cook T. Use of the laryngeal tube in 100 patients. Acta Anaesthesiologica Scandinavica 2003;47:828-32. [PubMed: 12859303]

15. Cook T, McKinstry C, Hardy R, Twigg S. Randomized crossover comparison of the Proseal laryngeal mask airway with the laryngeal tube during anaesthesia with controlled ventilation. British Journal of Anaesthesia 2003;91:678-83. [PubMed: 14570790]

16. Gaitini LA, Vaida SJ, Somri M, et al. An evaluation of the laryngeal tube during general anesthesia using mechanical ventilation. Anesthesia and Analgesia 2003;96:1750-5. [PubMed: 12761007]

17. Dorges V, Ocker H, Wenzel V, Schmucker P. The laryngeal tube: A new simple airway device. Anesthesia and Analgesia 2000;90:1220-2. [PubMed: 10781484]

18. Keller C, Brimacombe J. Pharyngeal mucosal pressures, airway sealing pressures, and fiberoptic position with the intubating versus standard laryngeal mask airway. Anesthesiology 1999;90:10016. [PubMed: 10201670]

19. Brimacombe J, Keller C, Brimacombe L. A comparison of the laryngeal mask airway Proseal and laryngeal tube airway in paralized anesthetized adult patients undergoing pressure-controlled ventilation. Anesthesia and Analgesia 2002;95:770-6. [PubMed: 12198070] 


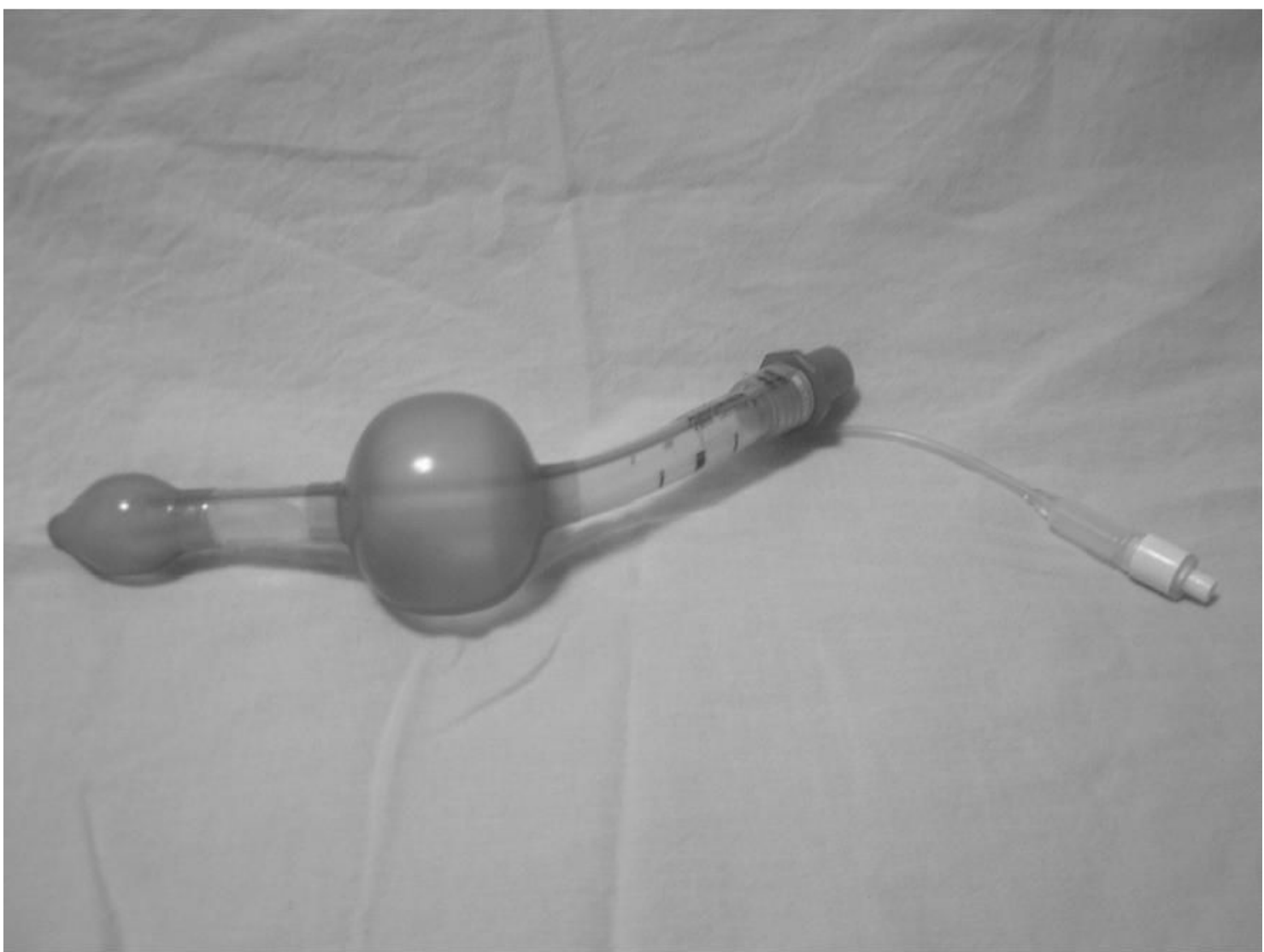

Figure 1.

Laryngeal tube. 


\section{Table 1}

Demographic and airway assessment data.

\begin{tabular}{lrc}
\hline Variable & N=51 \\
\hline & Age; years & $53(19)$ \\
& Sex; Male/Female & $16 / 35$ \\
Height; $\mathbf{~ m ~}$ & $159(8)$ \\
Weight; $\mathbf{k g}$ & $57(8)$ \\
& Mallampati score; $\mathbf{1 / 2 / 3}$ & $29 / 18 / 4$ \\
& Tnterincisor distance; cm & $4.7(0.6)$ \\
& Thyromental distance; cm & $7.5(0.8)$ \\
& Sternomental distance; cm & $15.8(1.5)$ \\
\hline
\end{tabular}

Data presented as mean (SD) or number of patients. 
Table 2

Assessment of device placement and ventilation.

\begin{tabular}{|c|c|c|c|}
\hline & LT & ILMA & $\boldsymbol{P}$ \\
\hline Insertion time; seconds & $28(23-35)[10-122]$ & $20(15-25)[10-65]$ & $<0.001$ \\
\hline \multicolumn{4}{|l|}{ Insertion attempts; number } \\
\hline - & 16 & 42 & $<0.001$ \\
\hline 2 & 20 & 9 & \\
\hline 3 & 7 & 0 & 0.005 \\
\hline Failures; number & 8 & 0 & 0.004 \\
\hline $\begin{array}{r}\text { Tidal volume at inspiratory pressure of } 20 \mathrm{~cm} \\
H^{2} \mathrm{O} ; \mathrm{ml}\end{array}$ & $440(290-670)[110-1230]$ & $630(440-750)[100-1050]$ & 0.013 \\
\hline Inspiratory pressure at gas leak; $\mathbf{c m ~ \mathbf { H } ^ { 2 } \mathrm { O }}$ & $24(21-30)[10-30]$ & $27(20-30)[10-30]$ & 0.44 \\
\hline $\begin{array}{r}\text { Gastric insufflation at inspiratory pressure } \\
20 \mathrm{cmH}^{2} \mathrm{O} \text {; number/total }\end{array}$ & $2 / 43$ & $5 / 51$ & 0.32 \\
\hline
\end{tabular}

Data presented as median (interquartile range) [range] or number. 